\title{
Out on the farm with DNA vaccines
}

\author{
Clayton W. Beard* and Peter W. Mason
}

Plum Island Animal Disease Center, Agricultural Research Service, United States Department of Agriculture, Greenport, NY 11944. ${ }^{*}$ Corresponding author (e-mail: cbeard@asrr.arsusda.gov)

Received 29 July 1998, accepted 22 October 1998

\begin{abstract}
DNA vaccination is a rapidly developing technology that offers new approaches for the prevention of disease. This technology may permit the production of new vaccines against diseases that have no current vaccine, as well as allowing the development of improved vaccines to replace existing products. We describe how DNA vaccination is being developed for use in commercial animal production, with an emphasis on viral diseases, and discuss the existing hurdles to its development and use.
\end{abstract}

Keywords: agricultural biotechnology, vaccine development, livestock/poultry

\begin{abstract}
Vaccination as a means of disease prevention in livestock/poultry production is essential for an economically sound and reliable food-animal industry. Vaccines can reduce the signs of disease, horizontal transmission of the infectious agent, and mortality. The reduction of horizontal transmission (herd immunity) is critical for the prevention of costly outbreaks of disease, which can devastate animal populations and interfere with global and national shipping and marketing. Vaccines are routinely used to control a number of economically important pathogens, and as new diseases emerge there is likely to be a need for the development of new vaccines.
\end{abstract}

\section{Vaccination and commercial animal production}

The life span of commercially raised animals presents a complex vaccination issue. In the United States, livestock maintained for meat production have short life spans: Beef cattle go to market after 18 months, swine at 6 months, lambs at 1-6 months, and chickens at 7-9 weeks. In order to be useful in meat-animal production, vaccination must not only be inexpensive but also promptly stimulate protective immunity. In contrast, other livestock, such as brood cows, dairy cows, breeder pigs, and racehorses, have life spans of many years and therefore require equally effective and inexpensive vaccines that elicit long-lasting immunity.

Usefulness of vaccines depends upon a favorable balance of efficacy, safety, and cost. Efficacy is defined as the ability of a vaccine to prevent mortality and morbidity, and to prevent replication of microorganisms, thereby reducing shedding and spread of the disease. A vaccine is considered safe if it does not cause disease or harmful side effects ${ }^{1}$. The cost of a vaccination is the sum of the costs of vaccine purchase, storage, administration, and any resulting loss of productivity. Since roundup of range animals is expensive and can result in loss of production due to stress, single-shot vaccines for multiple diseases are highly desirable. If the benefits of vaccination against a particular disease do not outweigh the risks and costs, culling diseased animals may be more efficient than vaccination. For some diseases, vaccination may be required by animal disease control agencies despite costs to individual producers.

Vaccines have traditionally consisted of killed microorganisms, subunits of microorganisms, or live attenuated microorganisms. Killed and subunit vaccines present a mass of preformed antigen to the immune system, which results in antibody production against components of the infectious agent. The methods used to derive these vaccines bias the immune response to specific subsets of immunogens that are presented to the host during a natural infection. Killed vaccines do not provide endogenously produced proteins needed to elicit cell-mediated immune responses and may require multiple inoculations to provide complete protection from infection.

Live attenuated vaccines produce immunogens in the recipient during a limited infection. In the case of viral vaccines, this results in production of antigens within the host animal's cells. These vaccines can cause clinical disease if they are not attenuated sufficiently or revert to more virulent forms. Administration of live attenuated vaccines may also be restricted in some cases, as young, pregnant, or immunocompromised animals cannot be vaccinated without significant risk of disease. In addition, young animals may have maternal antibodies that prevent replication of the attenuated microorganisms, eliminating or reducing the immune response to live attenuated vaccines. The production of large quantities of infectious material required for preparation of live attenuated or killed vaccines can present safety concerns due to the risk of escape of infectious agents or incomplete inactivation, especially if the agents are highly infectious or virulent.

The decision to vaccinate livestock/poultry depends on many factors, including openness of population, sanitation, and local disease threats. Currently, poultry are vaccinated against up to six diseases including Newcastle disease and infectious bronchitis. Beef cattle in the United States are vaccinated against as many as seven different pathogens including infectious bovine rhinotracheitis virus, bovine viral diarrhea virus, parainfluenza virus, and several Clostridium strains. Other vaccines may be used to combat outbreaks of diseases such as foot-and-mouth disease or classical swine fever (hog cholera). Although vaccination programs can be extremely beneficial, such as during management of epizootics or in disease eradication programs, vaccine-induced seroconversion can be confused with response to infection. Under these circumstances vaccination can complicate eradication or result in unnecessary condemnation. To circumvent this problem, "marker" vaccines have been created for several diseases (e.g., pseudorabies in swine $^{2}$ ) that permit differentiation between vaccinated and infected animals.

\section{DNA vaccines: Another step in vaccine development}

Other vaccine production strategies that include alternative methods to produce subunits, attenuate microorganisms, and develop attenuated microorganisms that serve as "vectors" to deliver subunit immunogens of other pathogens have been developed ${ }^{3}$. This latter type of vaccine strategy has been used to develop new livestock/poultry vaccines, although it has some limitations due to host immunity to the vector (including maternal antibodies) and 
its potential for causing disease. The demonstration that recombinant genes can be used to immunize animals in the absence of a biological vector (i.e., DNA vaccination) shows great promise as it provides many of the benefits of live attenuated vaccines without some of the risks and limitations.

The use of DNA as a vaccine was first proposed in 1990 in a report demonstrating that purified bacterial plasmid DNA ("naked" DNA) injected into the muscle of a mouse resulted in the expression of an encoded reporter gene $e^{4}$. These experiments suggested that a simple, "vectorless" vaccine could be created using a plasmid containing a cassette that included a eukaryotic promoter and a gene or genes encoding a protective antigen. When a DNA vaccine plasmid enters a eukaryotic cell, the proteins it encodes are transcribed and translated within the cell (Fig. 1). In the case of viral pathogens, these proteins are presented to the immune system in their native form, mimicking the presentation of antigens during a natural infection. However, the immune response to the expression of vaccine-encoded antigen may be affected by the location and type of cell expressing the antigen and the response may be manipulated by the addition of immunomodulator genes that are coexpressed with the vaccine plasmid.

Early DNA vaccine experiments used plasmids that encoded either the human growth hormone or the human $\alpha 1$-antitrypsin

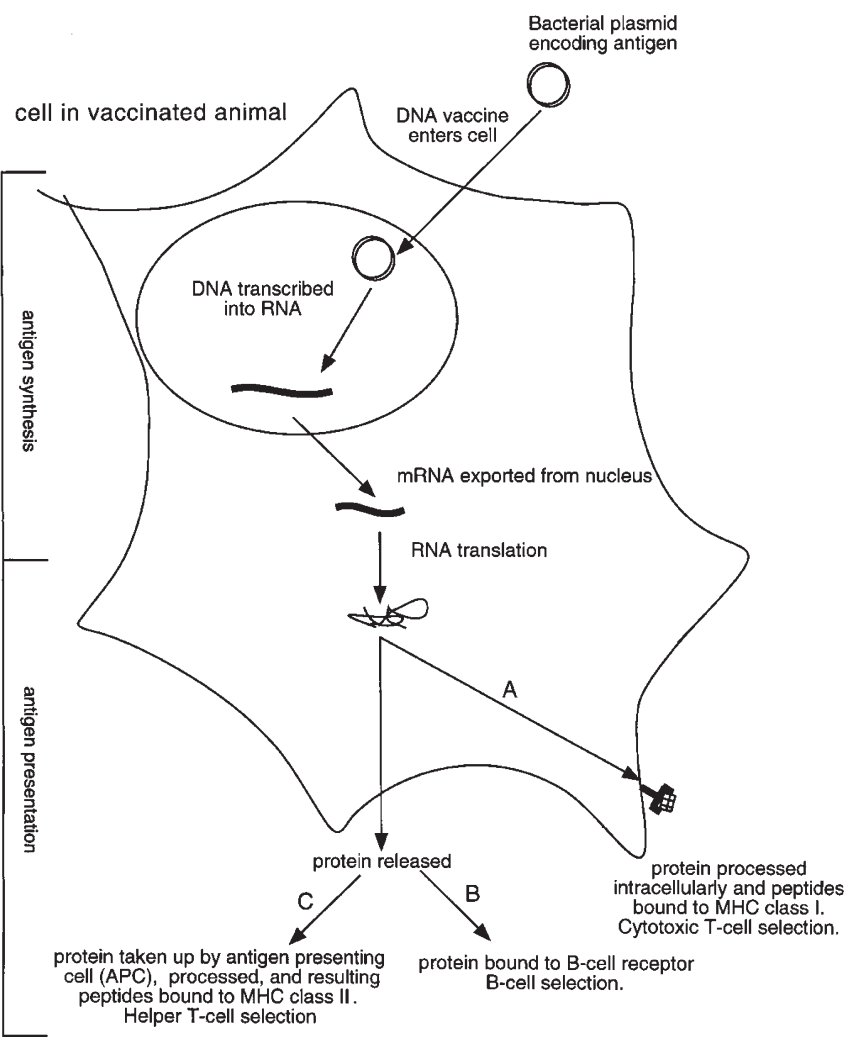

Figure 1. Schematic representation of steps required for immune response to DNA-encoded antigens. DNA plasmid enters cells of the inoculated animal and is transported into the nucleus. Transcribed RNA is translocated to the cytoplasm and translated, producing the protein antigen. The antigen is then presented to the immune system by several mechanisms. (A) The antigen is processed intracellularly and the resulting peptides are presented on the cell surface bound to major histocompatibility (MHC) class I molecules, where they serve in selection of cytotoxic T lymphocytes. (B) Protein is released (or secreted) from the cell where it can serve in selection of B cells. (C) Secreted protein is taken up by specific antigen presenting cells (including B cells), degraded, and its peptides are presented on the cell surface bound to MHC class II molecules, where they stimulates T-helper cells. gene under the control of either the human $\beta$-actin promoter or the cytomegalovirus (CMV) promoter. Mice inoculated with these plasmids developed antibodies against the encoded proteins $s^{5}$. Another early DNA vaccine encoded a hemagglutinin (HA) type 7 gene of influenza A virus under the control of the avian leucosis virus promoter. This vaccine was able to protect $50 \%$ of the inoculated chickens from lethal virus challenge ${ }^{6}$. The gene encoding a different influenza A polypeptide, the nucleocapsid protein, has also been inserted into DNA vaccine plasmids under the control of either the Rous sarcoma virus promoter or the CMV promoter. Following challenge, mice immunized with these plasmids showed increased survival rates, lowered lung virus titers, and reduced weight loss compared with unvaccinated controls ${ }^{7}$. Since these early reports, research on DNA vaccines has shown that the approach can be applied to many different pathogens and that there are multiple methods to alter and improve the immune response to these vaccines $^{8-17}$. Despite concerns that DNA vaccines may not be as effective as existing vaccines ${ }^{18}$, approximately a dozen DNA vaccines directed against a range of human diseases from AIDS to Tcell lymphoma are now in phase 1 clinical trials ${ }^{17}$.

DNA vaccines have several features that make them especially appealing (Table 1). They do not require cultivation of the infectious agent for their manufacture, so there is no danger of incomplete attenuation or of partial inactivation, as with live attenuated and killed vaccines. Furthermore, there is no risk of an agent mutating back to a virulent form or of causing disease in an immunocompromised animal. Therefore, DNA vaccines have the potential to be safer to use than existing inactivated or live attenuated vaccines. Moreover, DNA vaccine strategies may succeed for microorganisms that are difficult to cultivate or attenuate. The cost of producing plasmid DNA in bacteria is relatively low compared with the cost of producing infectious agents used for conventional vaccine manufacture. Once produced, DNA preparations are stable at room temperature, making these vaccines simpler and cheaper to store than many currently used vaccines. Significant production cost savings may also be possible for manufacturers making multiple vaccines because all DNA vaccines are likely to have similar production protocols. If DNA vaccines can be developed for multiple agents, it seems likely that they could be coadministered in a single dose, providing additional savings to users. As it appears likely that DNA vaccines will not pose any special threat to young or immunocompromised animals and that their immunogenicity will not be decreased by maternal antibodies, further flexibility in administration of these vaccines may be possible.

\section{State of development of DNA vaccines for commercial animals}

Livestock/poultry provide challenges in evaluation and development of vaccines due to the absence of well-characterized inbred

\section{Table 1. Advantages of DNA vaccines.}

- They do not require cultivation of dangerous infectious agents

- There is no risk of an attenuated vaccine strain mutating back to a virulent form

- They will not pose any special threat to young or immunocompromised animals, and immunogenicity will not be decreased by maternal antibodies

- They may be successful against microorganisms that are difficult to cultivate or attenuate

- There is a potential for low-cost production and administration

- They are inexpensive to store because they are stable at room temperature

- Vaccines can be developed for multiple agents and could be coadministered in a single shot 
Table 2. DNA vaccine trials in livestock/poultry.

\begin{tabular}{|c|c|c|c|c|c|}
\hline Species & Pathogen & Antigen & Antibody & Protection & Reference \\
\hline \multirow[t]{4}{*}{ Bovine } & Bovine herpes virus (BHV-1) & Glycoprotein D (gD) & Yes & $\begin{array}{l}\text { Reduced viral shedding and } \\
\text { Reduced clinical signs }\end{array}$ & 27 \\
\hline & $\mathrm{BHV}-1$ & Secreted gD & Yes & Yes & 26,28 \\
\hline & Bovine respiratory syncytial virus & G protein & Yes & Reduced viral shedding & 29,30 \\
\hline & Theileria annulata & $\begin{array}{l}\text { Surface antigens Tams } 1-1 \\
\text { and Tams1-2 }\end{array}$ & No & Partial & 32 \\
\hline \multirow[t]{5}{*}{ Porcine } & Influenza virus & Hemmaglutinin $(\mathrm{HA})$ & Yes & $\begin{array}{l}\text { accelerated clearance and } \\
\text { reduced shedding }\end{array}$ & 33 \\
\hline & Pseudorabies virus (PrV) & Glycoprotein C (gC) & Yes & Partial & 22 \\
\hline & PrV & Glycoprotein D (gD) & Yes & No & $19-21$ \\
\hline & $\begin{array}{l}\text { Foot-and-mouth disease } \\
\text { virus (FMDV) }\end{array}$ & $\begin{array}{l}\text { Replicating attenuated viral } \\
\text { geNome }\end{array}$ & Yes & Partial & 34 \\
\hline & FMDV & Virus capsid & Yes & Partial & 32 \\
\hline \multirow[t]{2}{*}{ Avian (chicken) } & Newcastle disease virus & F protein & Yes & Partial & 35 \\
\hline & Influenza virus & $\mathrm{HA}$ & Yes & Yes & 23 \\
\hline \multirow[t]{2}{*}{ Ovine } & Taenia ovis & Host-protective antigen (45W) & Low & ND & 36 \\
\hline & Cryptosporidium parvum & Surface antigen & Yes & ND & 37 \\
\hline
\end{tabular}

ND: Not determined

animal populations and the limited number of immunological reagents. In addition, livestock and poultry are more expensive and more difficult to work with than experimental laboratory animals. Despite these difficulties, encouraging progress has been made in the development of DNA vaccines for commercial animals.

As with DNA vaccines tested in laboratory animals, DNA vaccines in livestock/poultry have yielded various levels of protection from disease (Table 2). Several studies have tested the ability of pseudorabies virus (PrV) glycoprotein $\mathrm{D}(\mathrm{gD})$ to protect neonatal swine from Aujeszky's disease. These studies failed to show protection, and surprisingly, they have shown that colostrum-derived antibodies can reduce immune responses to DNA vaccines ${ }^{19-21}$.

Gerdts et al. ${ }^{22}$ tested two different DNA vaccines for Aujeszky's disease, one encoding the $\operatorname{PrV}$ envelope glycoprotein $\mathrm{C}(\mathrm{gC})$ and the other encoding gD. Using vaccines delivered intramuscularly, they found that the $\mathrm{gC}$ construct partially protected swine from lethal challenge whereas the gD-expressing construct did not. Further tests with the gC-based DNA vaccine showed that intradermal delivery with an injector gun was better than either intradermal injection or intramuscular injection. Moreover, pigs vaccinated three times using the intradermal injector were protected from challenge with lethal doses of PrV, and specific antibodies could be detected 9 months after vaccination.

Kodihalli et al. ${ }^{23}$ have developed a DNA vaccine that is superior to a conventional product in immunizing chickens against influenza. In their study, chickens were given two doses of either conventional (inactivated) influenza vaccine or a DNA vaccine encoding the HA (H5) protein under control of the cytomegalovirus promoter. Vaccinated birds were challenged with a homologous virus or antigenic variants. Both vaccines conferred similar high levels of protection from disease caused by homologous virus; however, the DNA vaccine conferred higher levels of protection from disease caused by heterologous virus.

\section{Future challenges}

There are several hurdles that need to be overcome on the road to the use of DNA vaccines on the farm. These include the technical challenges of improving delivery and/or potency so low doses of DNA can achieve the efficacy of conventional vaccines.

Use of DNA vaccines in commercial animals will also require the development of new regulations for their testing and use. The Center for Veterinary Biologics of the USDA and other regulatory agencies have begun to draft such guidelines to ensure the safety and efficacy of these vaccines. One of the safety issues surrounding the use of DNA vaccines is the potential for DNA integration into the genome of the host. To date, no experimental evidence has shown that integration can occur after DNA vaccination in mice ${ }^{24}$. DNA is routinely administered to humans and animals as a naturally occurring contaminant in many traditional vaccines. However, the specific introduction of large quantities of DNA has only been tested in a relatively small number of animals and even fewer humans. Another safety concern regarding use of DNA vaccines is the inadvertent stimulation of anti-DNA antibodies, which could cause autoimmune disease. Investigations into this issue have found no evidence of production of such antibodies ${ }^{25}$. During the testing of a potential DNA vaccine against bovine herpesvirus 1 , there were no anti-DNA antibodies detected 8 months after vaccination ${ }^{26}$. These findings provide a level of confidence that DNA vaccines will be safe to use in animals with a longer life span than laboratory mice. However, numerous other concerns must be answered before DNA vaccines can gain wide acceptance.

DNA vaccines hold great promise for improving food-animal production, especially in areas where pathogens have eluded traditional vaccine or therapeutic intervention. Improved efficacy, driven by continued research, and wide public acceptance will be required for the potential of DNA vaccines to be fully realized.

\section{Acknowledgments}

We thank Alfonzo Torres (PIADC) and Charles Beard (US Poultry and Egg Association) for helpful comments during the preparation of this article.

1. van Oirschot, J.T. 1994. Vaccination in food animal populations. Vaccine 12:415-418.

2. Mengeling, W.L., Brockmeier, S.L., Lager, K.M., and Vorwald, A.C. 1997. The role of biotechnologically engineered vaccines and diagnostics in pseudorabies (Aujeszky's disease) eradication strategies. Vet. Microbiol. 55:49-60.

3. Rolph, M.S. and Ramshaw, I.A. 1997. Recombinant viruses as vaccines and immunological tools. Curr. Opin. Immunol. 9:517-524.

4. Wolff, J.A., Malone, R.W., Williams, P., Chong, W., Acsadi, G., Jani, A. et al. 1990. Direct gene transfer into mouse muscle in vivo. Science 247:1465-1468.

5. Tang, D.C., De Vit, M., and Johnston, S.A. 1992. Genetic immunization is a simple method for eliciting an immune response. Nature 356:152-154.

6. Robinson, H.L., Hunt, L.A., and Webster, R.G. 1993. Protection against a lethal influenza virus challenge by immunization with a haemagglutinin-expressing 
plasmid DNA. Vaccine 11:957-960.

7. Ulmer, J.B., Donnelly, J.J., Parker, S.E., Rhodes, G.H., Felgner, P.L., Dwarki, V.J. et al. 1993. Heterologous protection against influenza by injection of DNA encoding a viral protein. Science 259:1745-1749.

8. Babiuk, L.A., Lewis, P.J., van Drunen Little-van den Hurk, S., Tikoo, S., and Liang, X. 1998. Nucleic acid vaccines: veterinary applications. Curr. Top. Microbiol. Immunol. 226:90-106.

9. Davis, H.L. 1998. DNA-based immunization against hepatitis B: experience with animal models. Curr. Top. Microbiol. Immunol. 226:57-68.

10. Fazio, V.M. 1997. "Naked" DNA transfer technology for genetic vaccination against infectious disease. Res. Virol. 148:101-108.

11. Levitsky, H.I. 1997. Accessories for naked DNA vaccines [news]. Nat. Biotechnol. 15:619-620.

12. Liu, M.A., McClements, W., Ulmer, J.B., Shiver, J., and Donnelly, J. 1997. Immunization of non-human primates with DNA vaccines. Vaccine 15:909-912.

13. Montgomery, D.L., Ulmer, J.B., Donnelly, J.J., and Liu, M.A. 1997. DNA vaccines. Pharmacol. Ther. 74:195-205.

14. Ramsay, A.J., Ramshaw, I.A., and Ada, G.L. 1997. DNA immunization. Immunol. Cell Biol. 75:360-363.

15. Robinson, H.L. 1997. Nucleic acid vaccines: an overview. Vaccine 15:785-787.

16. Simmonds, R.S., Shearer, M.H., and Kennedy, R.C. 1997. DNA vaccines-from principle to practice. Parasitology Today 13:328-331.

18. Manickan, E., Karem, K.L., and Rouse, B.T. 1997. DNA vaccines-a modern gimmick or a boon to vaccinology? Crit. Rev. Immunol. 17:139-154

19. Le Potier, M.F., Monteil, M., Houdayer, C., and Eloit, M. 1997. Study of the delivery of the gD gene of pseudorabies virus to one-day-old piglets by adenovirus or plasmid DNA as ways to by-pass the inhibition of immune response by colostral antibodies. Vet. Microbiol. 55:75-80.

20. Monteil, M., Le Potier, M.F., Guillotin, J., Cariolet, R., Houdayer, C., and Eloit, M. 1996. Genetic immunization of seronegative one-day-old piglets against pseudorabies induces neutralizing antibodies but not protection and is ineffective in piglets from immune dams. Vet. Res. 27:443-452.

21. Monteil, M., Le Potier, M.F., Cariolet, R., Houdayer, C., and Eloit, M. 1997. Effective priming of neonates born to immune dams against the immunogenic pseudorabies virus glycoprotein gD by replication-incompetent adenovirusmediated gene transfer at birth. J. Gen. Virol. 78:3303-3310.

22. Gerdts, V., Jons, A., Makoschey, B., Visser, N., and Mettenleiter, T.C. 1997. Protection of pigs against Aujeszky's disease by DNA vaccination. J. Gen. Virol. 78:2139-2146

23. Kodihalli, S., Haynes, J.R., Robinson, H.L., and Webster, R.G. 1997. Cross-protection among lethal H5N2 influenza viruses induced by DNA vaccine to the hemagglutinin. J. Virol. 71:3391-3396.

24. Nichols, W.W., Ledwith, B.J., Manam, S.V., and Troilo, P.J. 1995. Potential DNA vaccine integration into host cell genome. Ann. NY Acad. Sci. 772:30-39.

25. Mor, G., Singla, M., Steinberg, A.D., Hoffman, S.L., Okuda, K., and Klinman, D.M. 1997. Do DNA vaccines induce autoimmune disease? Hum. Gene Ther. 8:293-300.

26. van Drunen Littel-van den Hurk, S., Braun, R.P., Lewis, P.J., Karvonen, B.C., Baca-Estrada, M.E., Snider, M. et al. 1998. Intradermal immunization with a bovine herpesvirus-1 DNA vaccine induces protective immunity in cattle. J. Gen. Virol. 79:831-839.

27. Cox, G.J., Zamb, T.J., and Babiuk, L.A. 1993. Bovine herpesvirus 1: immune responses in mice and cattle injected with plasmid DNA. J.Virol. 67:5664-5667.

28. Braun, R., Babiuk, L., and van Drunen Littel-van den Hurk, S. 1997. Enhanced immune responses to an intradermally delivered DNA vaccine expressing a secreted form of BHV-1 gD. Vaccine Res. 6:151-164.

29. Schrijver, R.S., Langedijk, J.P., Keil, G.M., Middel, W.G., Maris-Veldhuis, M., Van Oirschot, J.T. et al. 1997. Immunization of cattle with a BHV1 vector vaccine or a DNA vaccine both coding for the G protein of BRSV. Vaccine 15:1908-1916.

30. Schrijver, R.S., Langedijk, J.P., Keil, G.M., Middel, W.G., Maris-Veldhuis, M., van Oirschot, J.T. et al. 1997. Comparison of DNA application methods to reduce BRSV shedding in cattle. Vaccine 16:130-134.

31. d'Oliveira, C., Feenstra, A., Vos, H., Osterhaus, A.D., Shiels, B.R., Cornelissen, A.W. et al. 1997. Induction of protective immunity to Theileria annulata using two major merozoite surface antigens presented by different delivery systems. Vaccine 15:1796-1804.

32. Chinsangaram, J., Beard, C.W., Mason, P.W., Zellner, M.K., Ward, G., and Grubman, M.J. 1998. Antibody response in mice inoculated with DNA expressing foot-and-mouth disease virus capsid proteins. J. Virol. 72:4454-4457.

33. Macklin, M.D., McCabe, D., McGregor, M.W., Neumann, V., Meyer, T., Callan, R et al. 1998. Immunization of pigs with a particle-mediated DNA vaccine to influenza a virus protects against challenge with homologous virus. J. Virol. 72:1491-1496.

34. Ward, G., Rieder, E., and Mason, P.W. 1997. Plasmid DNA encoding replicating foot-and-mouth disease virus genomes induces antiviral immune responses in swine. J. Virol. 71:7442-7447.

35. Sakaguchi, M., Nakamura, H., Sonoda, K., Hamada, F., and Hirai, K. 1996 Protection of chickens from Newcastle disease by vaccination with a linear plasmid DNA expressing the $F$ protein of Newcastle disease virus. Vaccine 14:747-752.

36. Rothel, J.S., Waterkeyn, J.G., Strugnell, R.A., Wood, P.R., Seow, H.F., Vadolas, J. et al. 1997. Nucleic acid vaccination of sheep: use in combination with a conventional adjuvanted vaccine against Taenia ovis. Immunol. Cell Biol. 75:41-46.

37. Jenkins, M., Kerr, D., Fayer, R., and Wall, R. 1995. Serum and colostrum antibody responses induced by jet-injection of sheep with DNA encoding a Cryptosporidium parvum antigen. Vaccine 13:1658-1664 